OPEN ACCESS

Edited by:

Wim Van den Ende,

KU Leuven, Belgium

Reviewed by:

Anja Thoe Fuglsang,

University of Copenhagen, Denmark

Alberto A. Iglesias,

National University of the Littoral,

Argentina

*Correspondence:

Paulo Arruda

parruda@unicamp.br

Specialty section:

This article was submitted to

Plant Physiology,

a section of the journal

Frontiers in Plant Science

Received: 13 March 2017

Accepted: 08 May 2017

Published: 26 May 2017

Citation:

Aquino B, Couñago RM, Verza N, Ferreira $L M$, Massirer $K B$, Gileadi $O$ and Arruda P (2017) Structural

Characterization of Maize SIRK1 Kinase Domain Reveals an Unusual Architecture of the Activation

Segment. Front. Plant Sci. 8:852. doi: 10.3389/fpls.2017.00852

\section{Structural Characterization of Maize SIRK1 Kinase Domain Reveals an Unusual Architecture of the Activation Segment}

\author{
Bruno Aquino ${ }^{1}$, Rafael M. Couñago ${ }^{1,2}$, Natalia Verza ${ }^{1,2}$, Lucas M. Ferreira', \\ Katlin B. Massirer, ${ }^{1,2}$, Opher Gileadi ${ }^{1,3}$ and Paulo Arruda ${ }^{1,2,4 *}$
}

' Structural Genomics Consortium, Universidade Estadual de Campinas, Campinas, Brazil, ${ }^{2}$ Centro de Biologia Molecular e Engenharia Genética, Universidade Estadual de Campinas, Campinas, Brazil, ${ }^{3}$ Structural Genomics Consortium, Nuffield Department of Clinical Medicine, University of Oxford, Oxford, United Kingdom, ${ }^{4}$ Departamento de Genética e Evolução, Instituto de Biologia, Universidade Estadual de Campinas, Campinas, Brazil

Kinases are primary regulators of plant metabolism and excellent targets for plant breeding. However, most kinases, including the abundant receptor-like kinases (RLK), have no assigned role. SIRK1 is a leucine-rich repeat receptor-like kinase (LRR-RLK), the largest family of RLK. In Arabidopsis thaliana, SIRK1 (AtSIRK1) is phosphorylated after sucrose is resupplied to sucrose-starved seedlings and it modulates the sugar response by phosphorylating several substrates. In maize, the ZmSIRK1 expression is altered in response to drought stress. In neither Arabidopsis nor in maize has the function of SIRK1 been completely elucidated. As a first step toward the biochemical characterization of $Z m S I R K 1$, we obtained its recombinant kinase domain, demonstrated that it binds AMP-PNP, a non-hydrolysable ATP-analog, and solved the structure of ZmSIRK1- AMPPNP co-crystal. The ZmSIRK1 crystal structure revealed a unique conformation for the activation segment. In an attempt to find inhibitors for $Z m S I R K 1$, we screened a focused small molecule library and identified six compounds that stabilized ZmSIRK1 against thermal melt. ITC analysis confirmed that three of these compounds bound to ZmSIRK1 with low micromolar affinity. Solving the 3D structure of ZmSIRK1-AMP-PNP co-crystal provided information on the molecular mechanism of ZmSIRK1 activity. Furthermore, the identification of small molecules that bind this kinase can serve as initial backbone for development of new potent and selective ZmSIRK1 antagonists.

Keywords: maize, receptor-like kinase, SIRK1, structure, ligand

\section{INTRODUCTION}

Kinases are thought to be key regulators of the mechanism by which plants respond to water restriction. Among kinases implicated in this process are some of the receptor-like kinases (RLK), which, at 610 members, comprise the largest kinase family in plants (Shiu and Bleecker, 2001). RLKs have also been implicated in innate immunity (Albert et al., 2010), pathogen response (Dodds and Rathjen, 2010), abiotic stress (Yang et al., 2010) and development and metabolism (De Smet et al., 2009). The largest sub-group of RLK possesses an extracellular domain containing leucine-rich repeats (LRR) (Shiu and Bleecker, 2001). These LRR-RLK are typically composed 
of a signal peptide, an extracellular domain with 1 to $30 \mathrm{LRR}$, a transmembrane domain and a cytoplasmic kinase domain. Crystal structures of LRR-RLK kinase domains revealed a canonical protein kinase fold and helped elucidate the cellular function of some LRR-RLK family members. For example, in the BIR2 structure access to the protein ATP-binding pocket was blocked by the $\beta 1$ strand. Furthermore, changes in the amino acid sequence of BIR2 P-loop, a conserved region in active kinases, prevented this protein from coordinating ATP properly. Together, these observations provided structural reasons for the inability of BIR2 to bind ATP and to function as a transferase (Blaum et al., 2014). The crystal structure of another LRR-RLK, BRI1, helped rationalize the observation that phosphorylation of amino acids in the protein activation segment could regulate BRI1 activity and increased its substrate specificity (Bojar et al., 2014).

Sucrose-induced receptor kinase 1 (SIRK1) is a member of the LRR-RLK family (Niittyla et al., 2007). In Arabidopsis, SIRK1 (AtSIRK1) is phosphorylated at residue S744 ${ }^{\text {AtSIRK1 }}$ after sucrose resupply to sucrose-starved plants and seems to be part of a signaling cascade regulating protoplast swelling and water uptake (Wu et al., 2013). AtSIRK1 is catalytically active and phosphorylates several substrates despite having amino acid substitutions at conserved positions important for kinase activity, including at the protein P-loop (GxGxxG; $\underline{G}$ to $S)$, activation segment (HRㅁKxxN; $\underline{D}$ to $\mathrm{N}$ ) and DFG (DYC) motifs (Wu et al., 2013).

Much less is known about the AtSIRK1 ortholog in maize, $Z m S I R K 1$. Expression profiling data reveals that the gene coding for ZmSIRK1 is down-regulated under drought stress and up-regulated after re-watering (Zheng et al., 2010); and is mainly expressed in the base of developing leaves (Sekhon et al., 2011; Stelpflug et al., 2016).

We have a long-term aim of elucidating the function of $Z m S I R K 1$ in drought stress by developing a chemical inhibitor that specifically targets its kinase domain. As a step toward this goal we cloned, expressed and purified the ZmSIRK1 kinase domain, identified several kinase inhibitors that bind to the enzyme and determined the first crystal structure of its kinase domain. We also identified small molecule kinase inhibitors that can bind to ZmSIRK1 and that might serve as starting points for the structure-guided development of more potent and selective inhibitors.

\section{MATERIALS AND METHODS}

\section{RNA Extraction and cDNA Synthesis}

Total RNA was extracted from leaves of 15-day-old maize B73 plants using PureLink RNA mini kit (Life Technologies, Carlsbad). Briefly, leaves were collected, immediately frozen in liquid nitrogen, and then powdered using a mortar. Two hundred $\mathrm{mg}$ of frozen powder was added to $1 \mathrm{~mL}$ of TRIzol (Invitrogen, Carlsbad). After $10 \mathrm{~min}$ incubation at room temperature with gentle shaking, $200 \mu \mathrm{L}$ of chloroform was added and the mixture was homogenized under vigorous agitation using a vortex. After centrifugation for $15 \mathrm{~min}$ at $12,000 \times g$ at $4^{\circ} \mathrm{C}$, the upper aqueous phase was collected and applied to a PureLink RNA (Life Technologies, Carlsbad) column. The column was washed with Wash buffer I followed by Wash buffer II and eluted in RNAse-free water as per the manufacturer's instructions. cDNA was synthesized using SuperScript III reverse transcriptase (Invitrogen, Carlsbad). Two microgram of total RNA were incubated with oligo dT20 and dNTP for $5 \mathrm{~min}$ at $65^{\circ} \mathrm{C}$ and then cooled on ice. Buffer, DTT and SuperScript enzyme were added and incubated for $60 \mathrm{~min}$ at $50^{\circ} \mathrm{C}$. The enzyme was inactivated for $15 \mathrm{~min}$ at $70^{\circ} \mathrm{C}$ and the $\mathrm{cDNA}$ stored at $-20^{\circ} \mathrm{C}$ until use.

\section{ZmSIRK1 cDNA Cloning and Protein Expression in Escherichia coli}

Four different N-terminal truncations of the ZmSIRK1 kinase domain (residues His623-Ser1045; Ser737-Ser1045; Ser751Ser1045; and Ser760-Ser1045) were cloned into pNIC28a-Bsa4 (Savitsky et al., 2010). Forward primers used were: sirk1-H623-F (TACTTCCAATCCATGCATTGGAAGATCAGTAGCTGGAAA AG); sirk1-S737-F (TACTTCCAATCCATGTCAGTTGTGTTCA CGGCTGAAG); sirk1-S751-F (TACTTCCAATCCATGTCT CCTGATAAACTGGTTGGGG); sirk1-S760-F (TACTTCCA ATCCATGTCTTGTGCCCCTGCTGAG) and reverse primer used was sirk1-S1045-R (TATCCACCTTTACTGTCACGAC GATAAGGACAAGAGATC). The cDNA synthesized from total RNA of maize B73 leaves was used as a template for PCR. After PCR amplifications, amplicons were treated with T4-DNA polymerase for ligase-independent cloning (LIC) (Savitsky et al., 2010). Positive clones for the four constructs were transformed into BL21(DE3)-R3-pRARE2 and grown in $500 \mu \mathrm{L}$ in $96 \times 1 \mathrm{~mL}$ well-block. After overnight growth, individual cultures were diluted 1:100 in LB medium with kanamycin $(50 \mu \mathrm{g} / \mathrm{mL})$ and incubated at $37^{\circ} \mathrm{C}$ with shaking. When the $\mathrm{OD}_{600}$ reached 2 , the temperature was lowered to $18^{\circ} \mathrm{C}, 0.1 \mathrm{mM}$ of IPTG was added and the culture incubated overnight with shaking. Cultures were then centrifuged at $3,500 \times g$ for $20 \mathrm{~min}$ at $4^{\circ} \mathrm{C}$ and lysed using $200 \mu \mathrm{L}$ of Lysis buffer (50 mM HEPES pH 7.5; $500 \mathrm{mM} \mathrm{NaCl}$; $10 \%$ glycerol; $10 \mathrm{mM}$ imidazole; $500 \mu \mathrm{M}$ TCEP; $0.1 \%$ dodecyl maltoside; $1 \mathrm{mM} \mathrm{MgCl} 2 ; 1: 200$ protease inhibitor; $0.5 \mathrm{mg} / \mathrm{mL}$ lysozyme; 50 units $/ \mathrm{mL}$ benzonase). After lysis, cultures were centrifuged at $3,500 \times g$ for $10 \mathrm{~min}$ at $4^{\circ} \mathrm{C}$ and the supernatant was incubated for $1 \mathrm{~h}$ at $18^{\circ} \mathrm{C}$ with $50 \mu \mathrm{L}$ of $\mathrm{Ni}^{2+}$-sepharose beads (GE Healthcare, Uppsala). After washing with wash buffer (50 mM HEPES pH 7.5; $500 \mathrm{mM} \mathrm{NaCl;} 10 \%$ glycerol; $30 \mathrm{mM}$ imidazole; $500 \mu \mathrm{M}$ TCEP), purified proteins were eluted with $50 \mu \mathrm{L}$ of $50 \mathrm{mM}$ HEPES pH 7.5; $500 \mathrm{mM} \mathrm{NaCl} ; 10 \%$ glycerol; $300 \mathrm{mM}$ imidazole; $500 \mu \mathrm{M}$ TCEP. Expression and solubility were verified in $12.5 \%$ SDS-PAGE (Laemmli, 1970).

\section{Large-Scale Protein Production and Purification}

Vector pNIC28a-Bsa4 harboring construct ZmSIRK1 $1^{737-1045}$ was transformed into competent BL21(DE3)-R3-pRARE2 Escherichia coli cells. Pre-culture was grown in $20 \mathrm{ml}$ of LB media grown overnight and then inoculated into $1.5 \mathrm{~L}$ of Terrific Broth at $37^{\circ} \mathrm{C}$ until $\mathrm{OD}_{600}$ of 1.5 . The culture was cooled down to $18^{\circ} \mathrm{C}$, $0.2 \mathrm{mM}$ of IPTG was added to the medium and growth resumed 
overnight. Cells were collected by centrifugation (15 min at $7,500 \times g$ at room temperature). Cell pellet was suspended in $2 \times$ binding buffer $(1 \times$ binding buffer is $500 \mathrm{mM}$ HEPES; $500 \mathrm{mM}$ $\mathrm{NaCl}$; $\%$ glycerol; $10 \mathrm{mM}$ imidazole; $1 \mathrm{mM}$ TCEP) with protease inhibitors (1:200) and frozen at $-80^{\circ} \mathrm{C}$ until use. Suspended cell pellets were thawed and sonicated for $9 \mathrm{~min}$ at $4^{\circ} \mathrm{C}(5 \mathrm{~s}$ ON; 10 s OFF; Amp 30\%). One $\mathrm{ml}$ of 5\% polyethyleneimine $(\mathrm{pH} 7.5)$ was added per $30 \mathrm{ml}$ of lysate and the sample was centrifuged at $53,000 \times g$ for $45 \mathrm{~min}$ at $4^{\circ} \mathrm{C}$. The supernatant was loaded onto an IMAC column ( $5 \mathrm{ml}$ HisTrap FF Crude) and washed in binding buffer with $30 \mathrm{mM}$ imidazole. Recombinant protein was eluted with elution buffer (binding buffer with $300 \mathrm{mM}$ imidazole). To remove the $6 \mathrm{xHis}$-tag, eluted protein was incubated with TEV protease and the tag removed using nickel beads. The protein solution was loaded onto a size exclusion HiLoad 16/60 Superdex 200pg (GE) column equilibrated in a gel filtration buffer (binding buffer without imidazole). Fractions of $1.8 \mathrm{~mL}$ were collected and verified for protein purity in a 12.5\% SDS-PAGE gel. Purified fractions were pooled together and stored at $-80^{\circ} \mathrm{C}$.

\section{Crystallization, Data Collection, Structure Determination and Refinement}

A mixture containing equimolar quantities of adenylylimidodiphosphate (AMP-PNP) and $\mathrm{MgCl}_{2}$ was added to purified ZmSIRK1 ${ }^{737-1045}(850 \mu \mathrm{M})$ at threefold molar excess. The solution was incubated on ice for approximately $30 \mathrm{~min}$. The mixture was centrifuged at $20,000 \times g$ for $10 \mathrm{~min}$ at $4^{\circ} \mathrm{C}$ prior to setting up $150 \mathrm{nl}$ volume sitting drops at three ratios of the protein-inhibitor complex to reservoir solution $(2: 1,1: 1$, or 1:2). Crystallization experiments were performed at $20^{\circ} \mathrm{C}$. The best-diffracting crystals grew under the conditions described in Table 1, first identified from the Morpheus Crystallization screen (Gorrec, 2015). Crystals were cryoprotected in reservoir solution supplemented with 20-25\% glycerol before flash-freezing in liquid nitrogen for data collection. Diffraction data were collected at the Advanced Photon Source (APS), integrated using XDS (Kabsch, 2010) and scaled using AIMLESS from the CCP4 software suite (Winn et al., 2011). Molecular replacement (MR) was performed with Phaser (McCoy et al., 2007) using the kinase domain of BAK1 interacting RLK 2 (BIR2) as the search model (PDB ID 4L68) (Blaum et al., 2014). Automated model building was performed with Buccaneer (Cowtan, 2006) following density modification with Parrot (Zhang et al., 1997). Automated refinement was performed in Buster (Global Phasing Ltd.). Coot (Emsley et al., 2010) was used for manual model building and refinement. Structure validation was performed using MolProbity (Chen et al., 2010). Structure factors and coordinates (Table 1) have been deposited in the PDB.

\section{Differential Scanning Fluorimetry (DSF)}

Thermal stabilization (DSF) assays were performed essentially as described (Niesen et al., 2007), with the following modifications. Purified ZmSIRK1 $1^{737-1045}$ was screened against a library of 378 structurally diverse and cell-permeable ATP-competitive kinase inhibitors library from Selleckchem (Houston, TX, United States; catalog No. L1200). DSF experiments were performed in a 384-well plate format. Each well contained $25 \mu \mathrm{L}$ of $1 \mu \mathrm{M}$ kinase in DSF buffer (100 mM K-phosphate, $150 \mathrm{mM} \mathrm{NaCl}$, $10 \%$ glycerol, $\mathrm{pH}$ 7.5) and the Protein Thermal Shift dye at the recommended concentration of 1:1000 (Applied Biosystems; the composition of the buffer and the dye solutions are not disclosed). Compounds $(10 \mathrm{mM})$ in DMSO were added to $10 \mu \mathrm{M}$ final concentration ( $0.1 \%$ final DMSO). Plates were sealed using optically clear films and transferred to a QuantStudio 6 qPCR instrument (Applied Biosystems, Singapore). Fluorescence intensity data were acquired in a temperature gradient from 25 to $95^{\circ} \mathrm{C}$ at a constant rate of $0.05^{\circ} \mathrm{C} / \mathrm{s}$ and protein melting temperatures were calculated based on a Boltzmann function fitting to experimental data, as implemented in the Protein Thermal Shift Software (Applied Biosystems, Singapore). Protein in $0.1 \%$ DMSO was used as a reference. Compounds displaying a positive temperature shift $(\Delta \mathrm{Tm})$ of $2^{\circ} \mathrm{C}$ or higher compared to the control were considered positive.

\section{Isothermal Calorimetry (ITC)}

Solutions containing $50 \mu \mathrm{M}$ purified $Z m$ SIRK1 $1^{737-1045}$ protein (cell) and $1 \mathrm{mM}$ AMP-PNP (injectant) were prepared in ITC buffer (50 mM K-phosphate; $500 \mathrm{mM} \mathrm{NaCl}$, 5\% glycerol; $1 \mathrm{mM}$

\section{TABLE 1 | Crystallographic data.}

\section{Data collection}

X-ray source

Wavelength $(\AA)$

APS 24-ID-C

Space group

Cell dimensions $(\AA)$

a, $b, c(\AA)$

Resolution $(\AA)$ *

No. of unique reflections*

$R_{\text {merge }}(\%)^{*}$

Mean $|/ \sigma|^{*}$

Completeness (\%)*

Redundancy*

$\mathrm{CC}_{1 / 2}{ }^{\ddagger}$

0.9790

$\mathrm{P} 2{ }_{1} 2{ }_{1} 2_{1}$

Refinement

Resolution range $(\AA)$

$R / R_{\text {free }}(\%)$

Mean B-factor (Å)

53.474 .579 .7

39.9-2.3

$57,012(1,084)$

$4.9(55.1)$

14.8 (2.6)

$97.0(98.4)$

$4.0(4.1)$

$0.99(0.91)$

39.8-2.3

$18.2 / 21.1$

66.9

0.010

1.06

Ramachandran plot statistics (\%)

Preferred regions

Outlier

PDB ID

Crystallization conditions

\section{2 \\ 0.0 \\ 5UV4}

12,5\% PEG1000; 12,5\% PEG3350; 12,5\% MPD; 0.02M of each D-glucose, D-mannose, D-galactose, L-fucose, D-xylose and $\mathrm{N}$-acetyl-D-glucosamine; 0.1 M MOPS/HEPES-Na pH7.5

*The highest resolution shell is shown in parenthesis. ${ }^{\ddagger} \mathrm{CC}_{1 / 2}$ indicates the percentage of correlation between intensities of random half data sets (Karplus and Diederichs, 2012). §r.m.s.d., root mean squared deviation from ideal geometry. 
TCEP; $50 \mathrm{mM} \mathrm{MgCl}_{2}$ ). For ITC measurements, $50 \mu \mathrm{M}$ of each compound (cell) and $500 \mu \mathrm{M}$ purified $Z m$ SIRK1 ${ }^{737-1045}$ (injectant) were prepared in ITC buffer without $\mathrm{Mg}^{2+}$. ITC experiments were performed in MicroCal AutiITC200 (GE, Northampton) and titrations were carried out at $25^{\circ} \mathrm{C}$ with a stirring speed of $750 \mathrm{rpm}$ and $300 \mathrm{~s}$ between each $2 \mu \mathrm{L}$ injection. Controls with buffer and ligand alone were performed to verify the dilution heat and were subtracted from protein-ligand data. Data were analyzed in Origin 7 software package.

\section{Phylogenetic Analyses}

The kinase domain of ZmSIRK1 was used to search related proteins within UniProt Knowledge database (e-value of $10^{-4}$; limit: 1,000 sequences). To verify the prevalence of putative phosphorylation sites within the activation segment of $Z m S I R K 1$ homologs we developed a script in java. The script performed a search within the identified proteins and reported on the presence/absence of the conserved DYS motif. If the DYS motif was present, then the script reported on the presence or absence of possible phosphorylation sites (Tyr, Ser, or Thr residues) within SIRK1 activation segment.

\section{RESULTS}

\section{Heterologous Expression and Purification of ZmSIRK1 Kinase Domain}

Constructs harboring the kinase domain of ZmSIRK1 were tested for recombinant expression in E. coli to obtain high yields of soluble protein. Four N-terminal truncations of the ZmSIRK1 cytoplasmic domain were designed and cloned into pNIC28a-Bsa4 (Figure 1A and Supplementary Figures S1A,B). Large-scale protein expression using construct $Z m S I R K 1^{737-1045}$ yielded $\sim 110 \mathrm{mg}$ of $\geq 98 \%$ pure recombinant protein using $1.5 \mathrm{~L}$ of culture (Supplementary Figures S1C-E). The identity of recombinant ZmSIRK1 kinase domain was verified by mass spectrometry (data not shown).

\section{Crystallization and Overall Structure of ZmSIRK1 Kinase Domain}

Crystallization conditions for purified ZmSIRK1 kinase domain were identified using commercially available sparse matrix crystallization screens, resulting in large ZmSIRK1-AMP-PNP co-crystals (Table 1 and Supplementary Figure S1F). The crystal structure of $Z m S I R K 1$ kinase domain bound to the non-hydrolysable ATP analog, AMP-PNP, was solved by molecular replacement using the kinase domain of AtBIR2, an RLK from Arabidopsis, as the search model (PDB ID 4L68; 35\% sequence identity) (Blaum et al., 2014) (Table 1). Overall, the ZmSIRK1 kinase domain has the canonical kinase topology, with an N-terminal lobe composed mostly of $\beta$-sheets connected by a flexible linker (hinge region) to a C-terminal lobe predominantly composed of $\alpha$-helices (Figure 2A). In the crystal structure, AMP-PNP is bound to the ATP-binding pocket of the ZmSIRK1 kinase domain, located in a cleft between $\mathrm{N}$ - and C-terminal lobes. Electron density maps allowed placement of most residues in ZmSIRK1. The final model consisted of residues 744-972, 977-1000, and 1009-1045. Omitted regions of the polypeptide chain in the final model located to the protein $\mathrm{N}$-termini or to loop regions in the C-terminal lobe, which are likely to be disordered due to flexibility.

\section{The Activation Segment of ZmSIRK1 Adopts an Unusual Conformation}

We compared the structure of ZmSIRK1 against those in the PDB using the DALI server (Holm and Rosenstrom, 2010). This search identified the kinase domains of AtBIR2 (BAK1-interacting RLK 2) and AtBSK8 (brassinosteroid signaling kinase 8 ) as the closest structural neighbors to $Z m S I R K 1$. Both AtBIR2 and AtBSK8 are known to be pseudokinases and to lack catalytic activity (Grutter et al., 2013; Blaum et al., 2014). The next closest structural neighbors to $Z m S I R K 1$ were the human protein kinase IRAK4 (interleukin-1 receptor-associated kinase 4) (Wang et al., 2006) and the kinase domain of the brassinosteroid insensitive membrane receptor from Arabidopsis (AtBRI1) (Bojar et al., 2014) (Figures 2A-E).

Compared to its closest structural neighbors, the most notable feature of $Z m$ SIRK1 kinase domain is the unusual architecture of its activation segment (highlighted in orange in Figure 2). The activation segment of kinases extends from the DFG $\left({ }^{907}\right.$ DYS $^{909}$ in $Z m S I R K 1)$ to the APE $\left({ }^{933} \mathrm{PPE}^{935}\right.$ in $\left.Z m S I R K 1\right)$ motifs. In most protein kinases, this region is composed of a flexible loop. The position of this loop controls the activations state of the enzyme. Kinases in a fully active state have activation segments in an extended or open conformation. By contrast, in the inactive kinase conformation, the position of the activation segment occludes the peptide-binding site. In ZmSIRK1, the activation segment is highly structured. The region immediately C-terminal to the DYS motif forms a single turn $3_{10}$ helix that packs against $\alpha \mathrm{C}$. This helical turn is followed by a short loop region and an $\alpha$-helix (P916 ${ }^{Z m S I R K 1}-{ }_{-L 929^{Z m S I R K 1}}$ ). The position and organization of the ZmSIRK1 activation segment are stabilized by hydrogen bonds to residues from both lobes of the protein (Figure 3A). Residue Gln922 within the $\alpha$-helix hydrogen bonds to residues in both the glycine-rich $\left(\mathrm{H} 772^{Z m S I R K 1}\right)$ and catalytic loops (G887 $\left.{ }^{Z m S I R K 1}\right)$. Moreover, Y $931^{Z m S I R K 1}$, located at the C-terminal end of the activation loop, hydrogen bonds to catalytic loop residue S891ZmSIRK1 and to E959 ${ }^{Z m S I R K 1}$ from $\alpha$-helix F (subdomain IX in PKA). These structures partly occlude the kinase ATP-binding site and peptide substrate-binding pocket, hallmarks of the inactive state (Figure 3B). An equivalent $3_{10}$ helix has been observed in the inactive state of mammalian kinases, such as ABL, SRC, and CDK2 (De Bondt et al., 1993; Schindler et al., 1999; Xu et al., 1999; Levinson et al., 2006). However, in these proteins, the $3_{10}$ helix is followed by a mostly disordered region, whereas in $Z m S I R K 1$ we observe a short loop followed by an $\alpha$-helix (Figure 3C). 
A

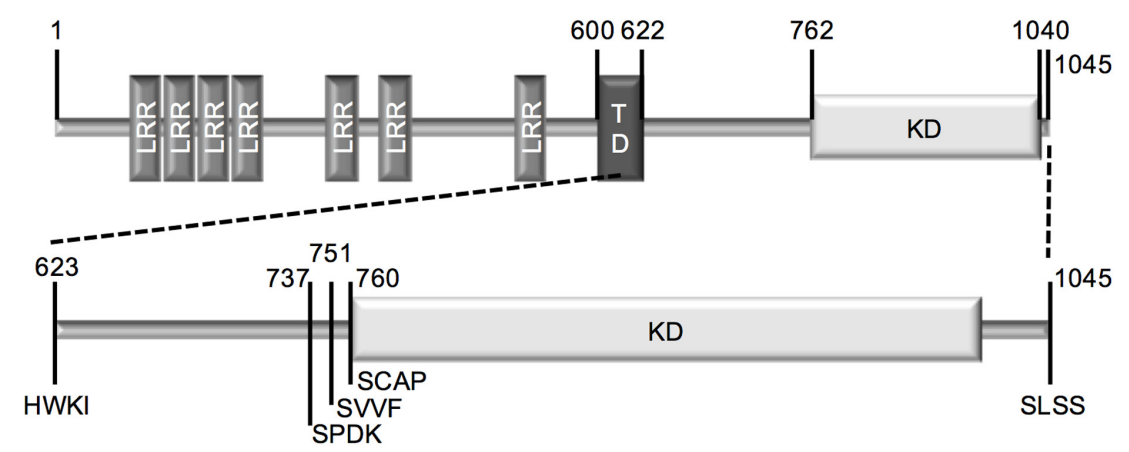

\section{B}

GxGxxG

SIRK1 Z, mays SIRK1 O.sativa A.thaliana 774 LGRSSHGT 781 BIR2 A.thaliana 313 IVSTRTGT 320 BRI1 A.thaliana 889 IGSGGFGD 896 SnRK3 A.thaliana 17 IGEGTFAK 24 BSK8 A.thaliana 65 HGERAPNV 72 IRAK4 H.sapiens 192 MGEGGFGV 199 PKA M.musculus 50 LGTGSFGR 57

\begin{tabular}{rll}
\multicolumn{3}{c}{ VAIK } \\
785 & MLTVKW & 790 \\
781 & ALTVKW & 786 \\
792 & MLTVKW & 797 \\
331 & ALAVKH & 336 \\
907 & AVAIKK & 912 \\
36 & NVAIKI & 41 \\
83 & RIAVKR & 88 \\
209 & TVAVKK & 214 \\
69 & HYAMKI & 74
\end{tabular}

$\mathbf{E}$

804 REI 806 800 REI 802 811 REA 813 349 YEM 351 926 AEM 928 58 REI 60 102 EEA 104

232 QEI 234 91 NEK 93
HRD K N 885 PHGNTKSSNVT 895 881 PHGNLKSSNVL 891 891 PHGNLKPTNII 901 426 LHQNICSSVIL 436 006 IHRDMKSSNVL 1016 131 YHRDLKPENLL 141 178 LYHDLNAYRVL 188 308 IHRDIKSANIL 318 164 IYRDLKPENLL 174

\begin{tabular}{cll}
\multicolumn{3}{c}{ DFG } \\
906 & TDYSL & 910 \\
906 & TDYSL & 910 \\
912 & TDYCV & 916 \\
446 & IDSGL & 450 \\
026 & SDFGM & 1030 \\
151 & SDFGL & 155 \\
198 & SCFGL & 202 \\
328 & SDFGL & 332 \\
184 & TDFGF & 188
\end{tabular}

FIGURE 1 | Cloning strategy for ZmSIRK1. (A) Schematic representation of full-length ZmSIRK1. The extracellular domain (aa 1-600) contains seven leucine-rich repeats (LRR) and is separated from the intracellular domain by a transmembrane domain (TD) (aa 601-622). The intracellular domain (aa 623-1045) contains a kinase domain (KD) (aa 762-1040). Numbers represents the amino acid position and letters the amino acid sequence from the beginning of each construct used for recombinant protein expression. (B) Amino acid sequence alignment of conserved motifs within ZmSIRK1 kinase domains with other protein kinases. GxGxxG: glycine-rich loop; VAIK: motif harboring the catalytic lysine; E: conserved glutamate in helix $\alpha \mathrm{C}$; HRDxKxxN: catalytic loop containing the catalytic aspartate; DFG: DFG motif.

\section{Conservation of Putative Phosphorylation Sites within ZmSIRK1 Activation Domain}

In most kinases, phosphorylation of regulatory Ser/Thr residues within the activation segment stabilizes the active state conformation of the kinase by counteracting an Arg residue within the HRD motif. Within the ZmSIRK1 activation segment, there are three putative phosphorylation sites (Figure 4A). The first, T915 ${ }^{\mathrm{ZmSIRK} 1}$, is located in the loop between the helical elements within the activation segment. The next two possible phosphorylation sites, Y931 ${ }^{Z m S I R K 1}$ and S932 ${ }^{Z m S I R K 1}$, are found immediately after the $\alpha$-helix. There are also three other possible phosphorylation sites immediately C-terminal to the activation segment (residues 937-940). Inspection of electron density maps did not suggest these residues are phosphorylated in the ZmSIRK1-AMP-PNP crystal structure. Moreover, the HRD motif in ZmSIRK1 is degraded and the positively charged Arg is replaced with a Gly. In some of ZmSIRK1 closest structural neighbors, both from plant and mammals, phosphorylation of Ser/Thr residues within the activation domain is required for activity (Hantschel and Superti-Furga, 2004; Wang et al., 2005; Cheng et al., 2007; Kuglstatter et al., 2007; Yan et al., 2012) (Figure 4B).

We reasoned that if these putative phosphorylation sites are involved in protein activation, they should be conserved amongst SIRK1 homologs. A search within UniProt using the kinase domain of $Z m$ SIRK1 revealed 124 proteins, exclusively from plants, bearing the unusual DYS motif present in maize SIRK1 (sequence identities between 29 and 98\%). We then interrogated these sequences for the presence of putative phosphorylation sites (Thr/Tyr/Ser residues) at equivalent positions within and immediately C-terminal of ZmSIRK1 activation segment -+6 , $+22,+23$, and $+29-31$ positions C-terminal from the DYS motif. We found that 119 out of 124 proteins with a DYS motif in Uniprot displayed a Thr residue at position +6 , which locates to the spacer loop between the $3_{10}$ and $\alpha$ helices within $Z m$ SIRK1 activation segment. We also found that all 124 proteins displayed a Tyr residue at positions +22 - located immediately C-terminal to the $\alpha$-helical segment in $Z m S I R K 1$; in addition to having a Thr residue at position +6 . Conservation at positions +29 (Ser), +30 (Ser/Thr), and +31 (Ser/Thr) was also high (ranging from 80 to $90 \%)$, whereas sites at +23 and +28 were markedly less conserved $(<21 \%)$. Overall, co-conservation of the most prevalent residues occurred in 79 out of the 124 identified proteins $(\sim 64 \%)$. These analyses also identified 209 plant SIRK1 homologs with the DYC motif seen in AtSIRK1. Conservation of possible phosphorylation sites within these proteins is similar, but not identical to those identified above for $Z m S I R K 1$ (Supplementary Table S1).

\section{ZmSIRK1 Adopts an Inactive, DFG-in $/ \alpha$ C-Out Conformation}

In many kinases, the positions of $\alpha \mathrm{C}$ and of the DFG motif in the active and inactive states are also different. In the active conformation, $\alpha \mathrm{C}$ swings inward toward the ATP-binding 
A

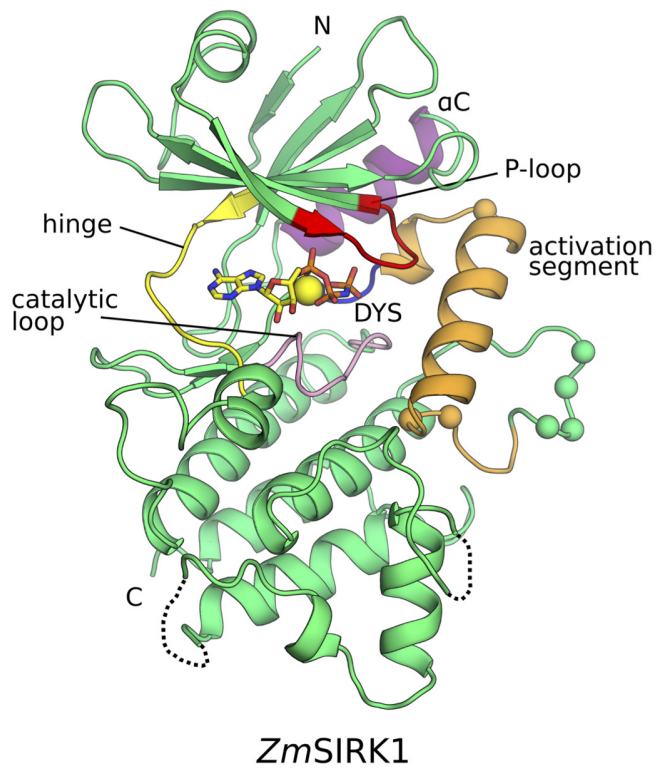

B

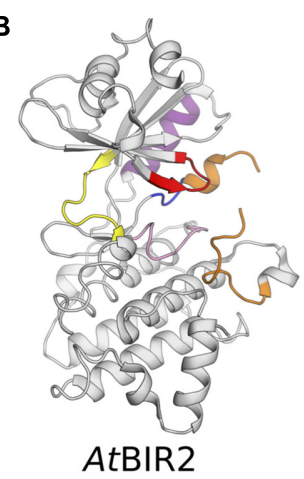

D

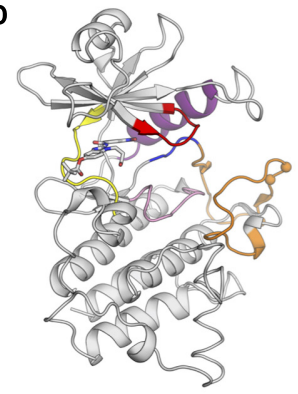

HsIRAK4
C

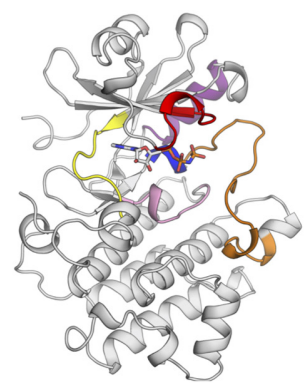

AtBSK8

E

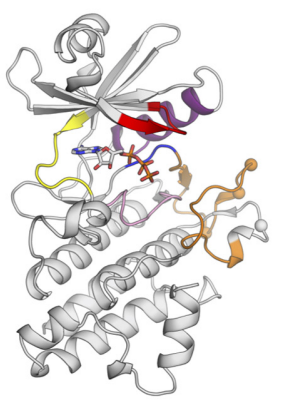

AtBRI1

FIGURE 2 | Co-crystal structure of ZmSIRK1 bound to AMP-PNP. (A) Cartoon representation of the ZmSIRK1-AMP-PNP structure. Protein regions were colored as follows: glycine-rich (P-)loop - red; hinge region - yellow; catalytic loop -pink; DYS (more commonly DFG) motif - blue; activation segment - orange; $\alpha \mathrm{C}$ - purple. Other protein regions in green. AMP-PNP is shown in stick and $\mathrm{Mg}^{2+}$ ion as a yellow sphere. The Cas from putative phosphorylation sites within and immediately C-terminal of ZmSIRK1 activation segment are shown as spheres. (B-E) Cartoon representation of ZmSIRK1 closest structural neighbors. (B) Crystal structure of AtBIR2 (PDB ID 4L68). (C) Crystal structure of AtBSK8 (PDB ID 4I92). (D) Crystal structure of HsIRAK4 (PDB ID 2NRY). (E) Crystal structure of AtBRI1 (PDB ID 5LPB). Protein regions colored as in (A). If present, ligands are represented as sticks and the Cas from known phosphorylation sites as spheres.
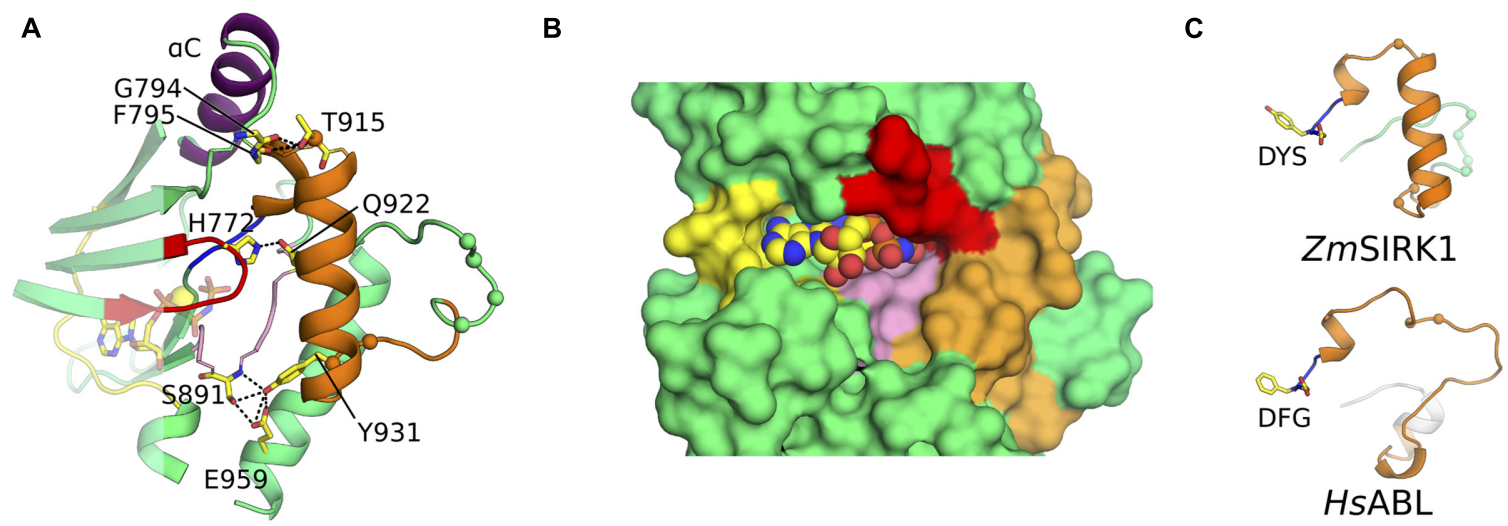

FIGURE $3 \mid$ ZmSIRK1 activation segment is highly structured. (A) Cartoon representation of ZmSIRK1 around the activation segment. Residues taking part in the stabilization of this segment are shown in stick. Possible hydrogen bonds are represented as dashed lines. (B) Surface representation of ZmSIRK1 nucleotide-binding site showing that the protein activation segment (orange) occludes the region normally available to interact with the target peptide. AMP-PNP is shown as spheres. (C) The 310 helix immediately C-terminal of ZmSIRK DYS motif (top) is also seen in inactive state mammalian kinase domains (bottom - human ABL; PDB ID 2G1T). The Cas from putative phosphorylation sites in ZmSIRK1 and from known phosphorylation sites in HsABL are shown as spheres.

pocket ( $\alpha$ C-in conformation) and facilitates the formation of a salt bridge between a conserved glutamic residue within $\alpha \mathrm{C}\left(\mathrm{E} 805^{Z m S I R K 1}\right)$ and the catalytic lysine $\left(\mathrm{K} 789^{Z m S I R K 1}\right)$. This interaction is key for enzyme activity. By contrast in the inactive $\alpha \mathrm{C}$-out conformation observed for a few mammalian proteins, this conserved glutamic residue is solvent-exposed and makes a salt link to an Arg residue from the kinase activation segment (Levinson et al., 2006). The interaction of the conserved aspartic 


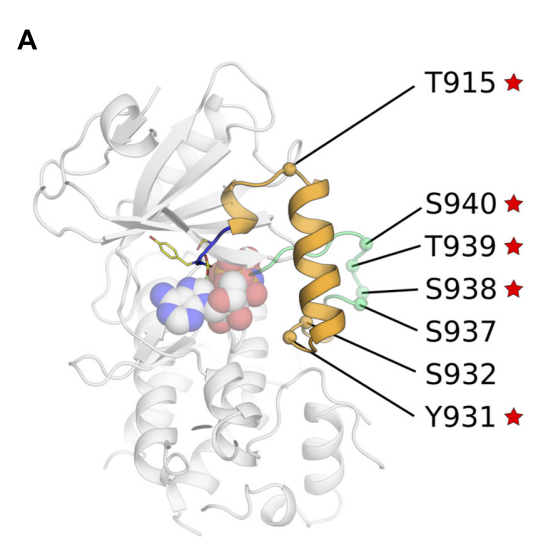

B

ZmS I RK 1

A tBIR2

At BSK 8

AtBRI 1

AtBAK 1

$H S C A B l$

HSIRAK 4

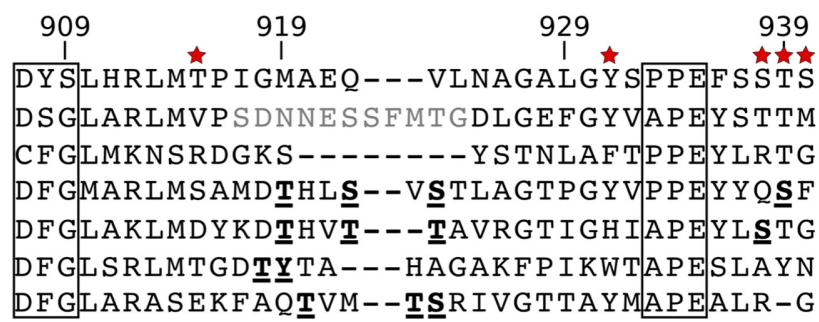

FIGURE 4 | Conservation of possible phosphorylation sites within ZmSIRK1 activation segment. (A) Cartoon representation of ZmSIRK1 highlighting the position of possible phosphorylation sites within (orange) and C-terminal (green) of the protein activation segment. Red stars indicate sites with high (> 80\%) conservation within SIRK1 homologs. (B) Structure-based sequence alignment of the activation segment in ZmSIRK and its closest structural neighbors. Conserved kinase domain motifs (commonly DFG and APE) are boxed. Residues in bold and underlined are known phosphorylation sites. Residues in gray were not visible in the structure.

residue within the DFG motif to the $\mathrm{Mg}^{2+}$ ion is also critical for transphosphorylase activity. This interaction is only possible in the so-called DFG-in conformation. In this configuration, the hydrophobic residue within the DFG motif takes part in a series of hydrophobic contacts that stabilizes the active state conformation of the enzyme (R-spine) (Kornev et al., 2006). In an active kinase, both DFG-in and $\alpha \mathrm{C}$-in configurations are usually present.

The kinase domain in the ZmSIRK1-AMP-PNP co-structure displays a DYS-in conformation and the side chain atoms from D $907^{Z m S I R K 1}$ within this motif participates in the octahedral coordination of the $\mathrm{Mg}^{2+}$ ion bound to AMP-PNP. However, coordination of the divalent ion is unusual (see below) and the position of $Y 908^{Z m S I R K 1}$ in the ZmSIRK1-AMP-PNP co-structure prevents assembly of the hydrophobic residues in the kinase domain core characteristic of active state proteins. The $\alpha \mathrm{C}$ in ZmSIRK1 is away from the ATP-binding site ( $\alpha \mathrm{C}$-out) and the side chain of E805 $5^{Z m S I R K 1}$ is solvent exposed and makes a hydrogen bond to R912 ${ }^{Z m S I R K 1}$ in the activation segment. Moreover, the hydroxyl group from Y908 ${ }^{Z m S I R K 1}$ hydrogen bonds to the main chain carbonyl group from $L 809^{Z m S I R K 1}$ in $\alpha \mathrm{C}$, further stabilizing the observed "twisted-out" configuration of this secondary structure (Figure 5A). The DFG-in/ $\alpha \mathrm{C}$-out out configuration has been observed for a few mammalian kinases, such as ABL, SRC, and CDK2 (Figure 5B), but thus far not in any plant kinase domains. For the mammalian kinases, phosphorylation of activations segment Ser/Thr residues can stabilize the active, DFG-in $/ \alpha \mathrm{C}$-in conformation (Figure $5 \mathrm{C}$ ).

\section{Interaction of ZmSIRK1 with AMP-PNP}

In the co-crystal structure, the ATP analog adopts an unusual conformation. The ribose is found in a $\mathrm{C} 2^{\prime}$-endo conformation, compared to the more commonly observed $3^{\prime}$-endo puckering. The position of the $\beta$-phosphate is also quite distinct and, consequently, so are the position and coordination of the $\mathrm{Mg}^{2+}$ ion (Figure 5D). The conformation observed for AMP-PNP here is similar to that in the inactive state structure of the mammalian protein CDK2 (Schulze-Gahmen et al., 1996) (Figure 5E) and contrasts with that observed for kinases in their active state, especially in what concerns the position of the nucleotide phosphate $\beta$ (Figure 5F).

The position of the ATP-analog within the ZmSIRK1 binding pocket is also unusual. The two lobes of a kinase domain are connected by a flexible "hinge" region. In ZmSIRK1, the hinge region spans residues S836-L844 ${ }^{Z m S I R K 1}$. In general, ATP and its analogs are anchored to the kinase domain via two hydrogen bonds. The adenosine N6 amine and N1 $\alpha \alpha$ imine interact with the backbone of residues located at +1 and +3 positions relative to the so-called gatekeeper residue (GK, S836 ${ }^{Z m S I R K 1}$ ), respectively. These interactions ensure the nucleotide sits deep within the enzyme binding pocket. However, in ZmSIRK1-AMP-PNP co-crystals, the N6 amine from the ligand interacts with the more solvent-exposed residue at $\mathrm{GK}+3\left(\mathrm{~A} 839^{\mathrm{ZmSIRK1}}\right)$. This is the only hydrogen bond between ligand and hinge residues. As a result, AMP-PNP sits closer to the solvent than it is normally observed for other kinases.

In most kinases, proper orientation of ATP phosphate groups within the kinase nucleotide-binding pocket relies on the close contacts afforded by glycine residues within the protein P-loop and is crucial for catalysis. As for other plant RLKs, the P-loop of ZmSIRK1 does not display the canonical consensus sequence (GxGxxG). In ZmSIRK1, the second Gly within this motif is replaced with S770 ${ }^{Z m S I R K 1}$. In the ZmSIRK1-AMP-PNP co-structure, the side chain from this serine residue hydrogen bonds to the $\gamma$-phosphate in AMP-PNP. The bulkier side chain of $S 770^{Z m S I R K 1}$ also prevents the nucleotide $\beta$-phosphate to interact closely with main chain groups from the P-loop. Instead, the phosphate groups from AMP-PNP interact mostly with protein residues in the protein $\mathrm{C}$-terminal domain, opposite to the $\mathrm{N}$-terminal P-loop. 


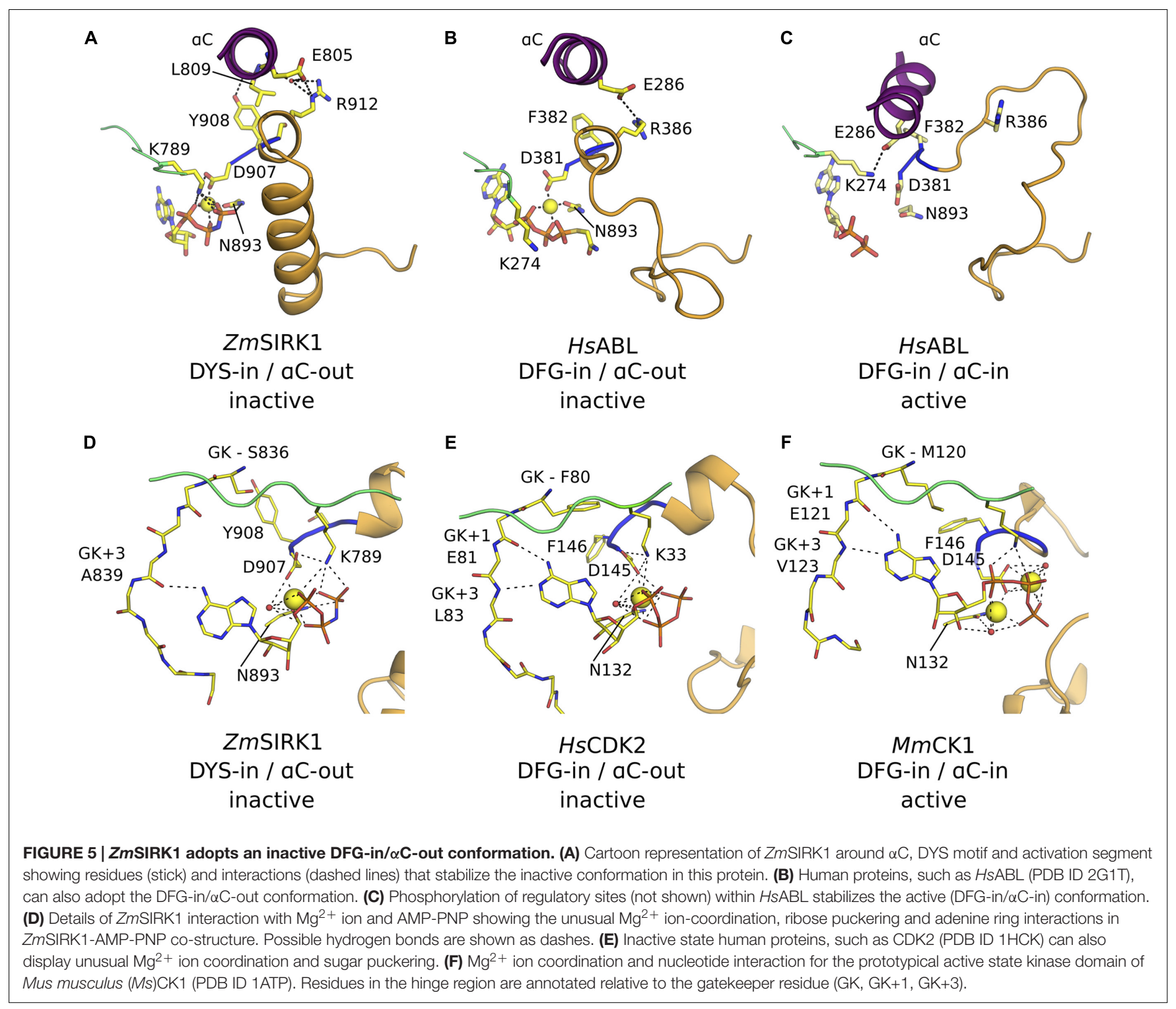

\section{Ligand Interaction}

In Arabidopsis, the expression of AtSIRK1 is induced by sucrose and the enzyme phosphorylates an aquaporin regulating water content inside cells. Identification of potent antagonists of ZmSIRK1 may help elucidate the role of this kinase during drought stress. Some of the closest structural homologs of ZmSIRK1 are pseudo-kinases, including BIR2 which does not bind ATP (Blaum et al., 2014). We thus determined if purified recombinant ZmSIRK1 could bind the non-hydrolysable ATP analog AMP-PNP using isothermal titration calorimetry (ITC). The ITC results indicated that the protein can bind the AMP-PNP in a magnesium-dependent manner with a $K_{\mathrm{D}}$ of $2.8 \mu \mathrm{M}$ (Figure 6).

We next used thermal denaturation assays (DSF) to identify small molecules within a 378-compound collection of chemically diverse human kinase inhibitors that could interact with ZmSIRK1. We identified three compounds with temperature shifts, $\Delta \mathrm{Tms}$, over $2.0^{\circ} \mathrm{C}$, an arbitrary cut-off for a positive hit in this experiment (Table 2). DSF results for these compounds were further confirmed using ITC. The compounds PP242, INK 128 and PP121 bound $Z m S I R K 1$ with $K_{\mathrm{D}} \mathrm{s}$ of $0.46,1.76$, and $1.99 \mu \mathrm{M}$, respectively (Table 2 and Supplementary Figure S2).

The ZmSIRK1-ATP-PNP co-structure showed a partly closed ATP-binding site. To assess if compounds identified by DSF and verified by ITC would be able to fit into the ATP-binding pocket of ZmSIRK1, we performed a simple rigid-body docking of PP121 to the ZmSIRK1-AMP-PNP co-structure. This exercise used the available co-structure of PP121 bound to the human kinase domain SRC (PDB ID 3EN4) (Apsel et al., 2008) and suggested PP121 can fit in the ATP-binding pocket of ZmSIRK1 and occupies the bottom of this cavity with few steric clashes to protein residues (Supplementary Figure S3). PP242 and INK 128 are closely related to PP121 (Table 2) and we expect they could also fit without major clashes in the nucleotide-binding pocket 


\section{Control}

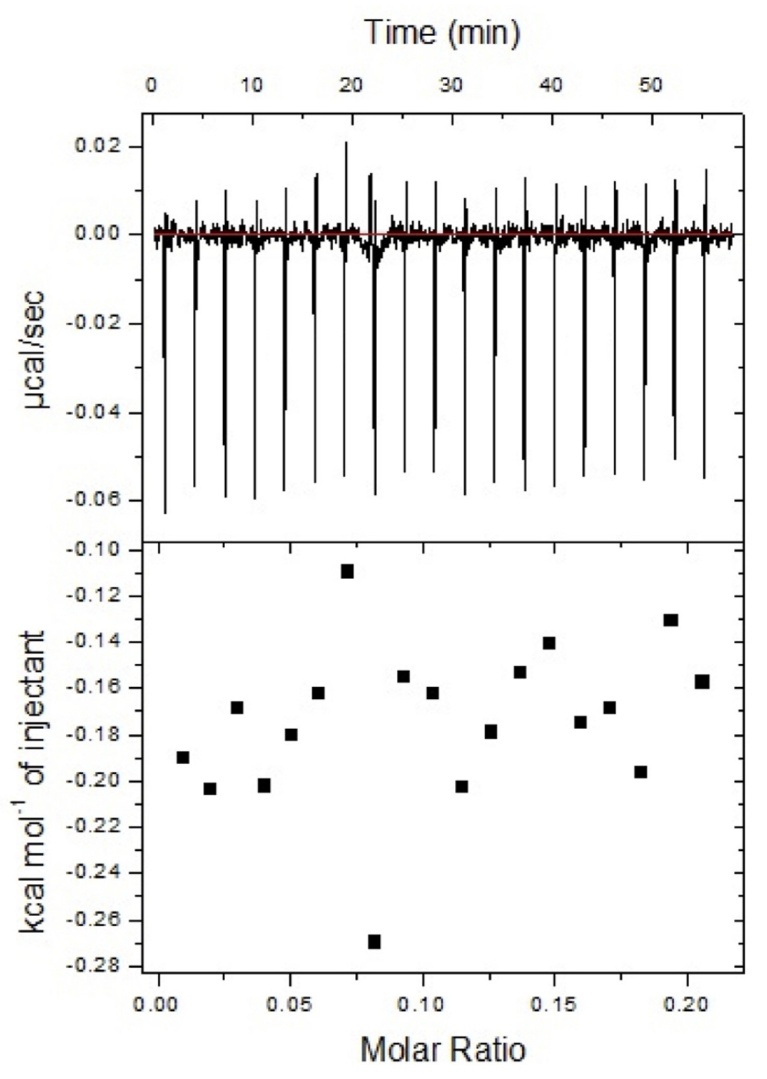

\section{AMP-PNP}

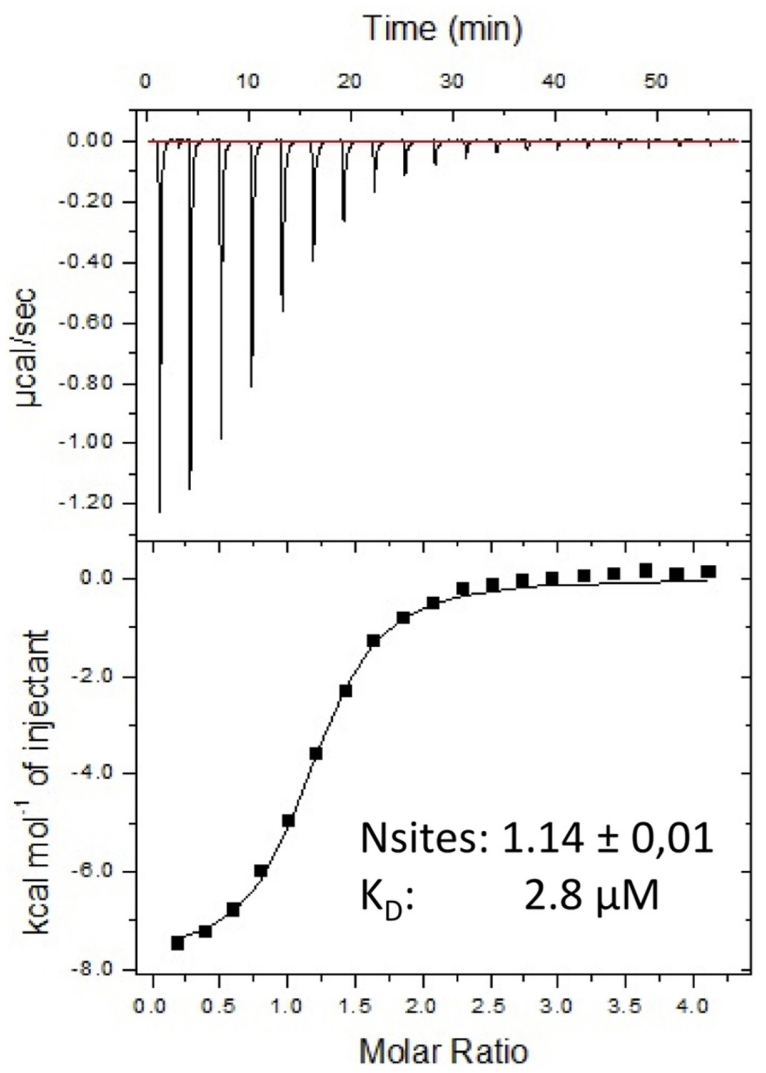

FIGURE 6 | ZmSIRK1 binds to AMP-PNP in solution. Isothermal titration calorimetry (ITC) measurements for the interaction between AMP-PNP and ZmSIRK1. Protein (50 $\mu \mathrm{M}$ of $\left.Z m S I R K 11^{737-1045}\right)$ was titrated to AMP-PNP (1 mM) in ITC buffer (50 mM K-phosphate; 500 mM NaCl, $5 \%$ glycerol; 1 mM TCEP; 50 mM MgCl $)$. Y-axis in left (control) and right (AMP-PNP) panels are not in the same scale.

of ZmSIRK1. We have not yet been able to obtain co-crystal structures with these synthetic ligands.

\section{DISCUSSION}

Extensive structural characterization of mammalian kinase domains has captured a number of proteins in both active and inactive states. Phosphorylation of regulatory sites within the kinase activation segment stabilizes the closed, active state of the protein - a conserved activating mechanism observed in most protein kinases. Activation of plant protein kinases also requires phosphorylation of regulatory sites within the activation segment. For example, AtBRI1 activation segment must be phosphorylated for the kinase to be active (Wang et al., 2005). However, the structural details of how plant kinases switch from inactive to active states have not yet been completely characterized. The structure of the $Z m S I R K 1$ kinase domain obtained here suggests a possible activation mechanism for this and related plant kinases.

The activation segment of ZmSIRK1 adopts an unusual conformation - a single-turn 310 helix connected to an $\alpha$-helix by a short loop. The $\alpha$-helix within the activation segment occupies the putative peptide substrate-binding pocket. The protein is in a DYS-in $/ \alpha \mathrm{C}$-out inactive conformation, reminiscent of that observed for the inactive state of mammalian proteins, such as SRC, ABL, and CDK2 (De Bondt et al., 1993; Schindler et al., 1999; Xu et al., 1999; Levinson et al., 2006). Activation of these kinases requires phosphorylation of regulatory sites, mostly located at the proteins activation segment. ZmSIRK1 has several conserved, putative phosphorylation sites within and immediately C-terminal to its activation segment. Some of these putative phosphorylation sites in SIRK1 are structurally equivalent to known phosphorylation sites in AtBRI1 that stabilize the activation segment of this protein in an extended, active conformation (Tang et al., 2008; Bojar et al., 2014). It is thus possible that phosphorylation of regulatory sites within ZmSIRK1 activation segment elicits the large conformational changes required for this region of the protein to adopt an extended conformation. These structural movements would re-position the short helical turn within the activation segment and allow $\alpha \mathrm{C}$ to swing inwards toward the ATP-binding site. The ZmSIRK1AMP-PNP co-crystal suggests that ligand binding alone is not sufficient to elicit these conformational changes. 
The use of small molecules to modulate protein function has been extremely successful in assigning gene function in animal cells (Arrowsmith et al., 2015). For human proteins, such as bromodomains and protein kinases, several potent and selective chemical probes are available (Bain et al., 2007; Shortt et al., 2017). A similar approach has only started to be applied to plants. For example, glycogen synthase kinase 3 (GSK3) activity is required for brassinosteroid signaling. A loss-of-function mutation in the gene encoding GSK3 BIN2, a known negative regulator of the brassinosteroid signaling pathway, is not sufficient to activate this pathway. Polyploidy and genome duplication is a common phenomenon in plants and gene redundancy can overcome the deleterious effects of having a non-functional copy of a particular gene (Comai, 2005). However, the small molecule bikinin, an inhibitor of BIN2 and other GSK3, can activate the brassinosteroid signaling pathway in a hormone-independent manner (Vert and Chory, 2006; De Rybel et al., 2009). Thus, small molecule inhibitors targeting closely related members of the same kinase family may be used to overcome the inherent genetic redundancy of plants.

The evolutionary distances and lifestyle differences between plants and animals are likely to be reflected in both sequence and structure of plant kinases. The primary sequence of SIRK1 differs from that of mammalian kinases in key regions of

TABLE 2 | Thermal shift (DSF) and ITC data for selected ZmSIRK1 137-1045 ligands.

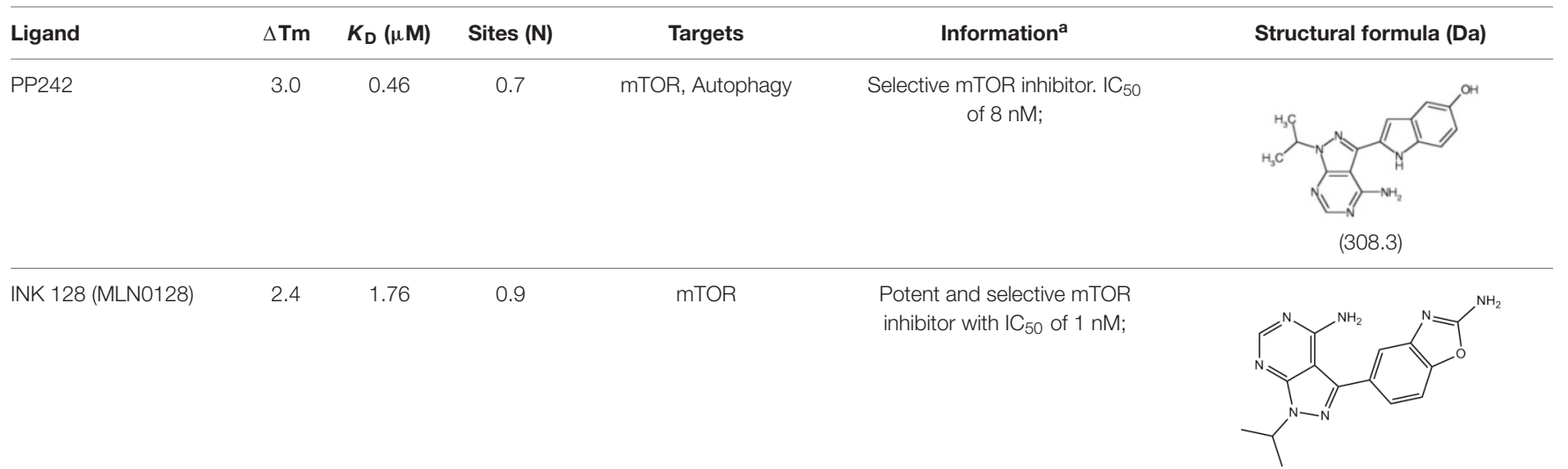

(309.3)

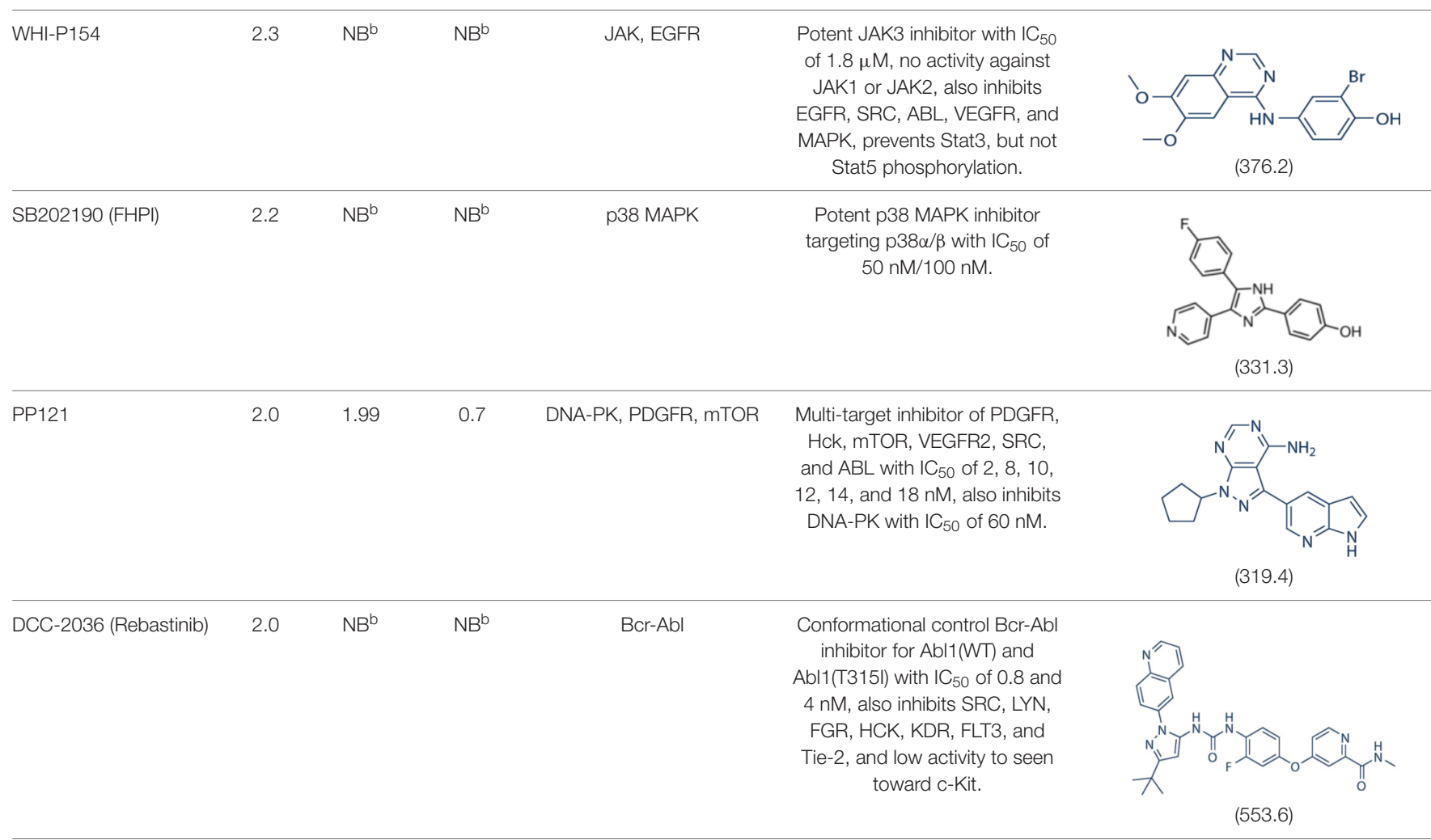

${ }^{a}$ Obtained from compound provider (Selleckchem). ${ }^{\mathrm{b}}$ Binding could not be confirmed by microcalorimetry. 
the protein, such as the activation segment, DFG/APE motifs and the P-loop. In many plant kinases, degradation of these conserved motifs has led to an inability to bind nucleotides (Blaum et al., 2014). However, we show here that ZmSIRK1 can bind to nucleotides and identified related synthetic compounds with similar chemotypes that can bind to ZmSRIK1 with low micromolar affinity, an indication that these molecules interact with a specific site within the protein, and that chemical libraries designed for human kinases will generate hits for some members of the plant kinome.

\section{CONCLUSION}

Here we report the first crystal structure of a SIRK1 kinase domain. Our results further suggest activation mechanism are conserved in both plant and mammalian protein kinases and that the plant enzymes can interact with compounds designed for their mammalian counterparts. Further work is required to elucidate the impact of phosphorylation on the structure and transphosphorylase activity of ZmSIRK1.

\section{ACCESSION}

Structure factors and coordinates were deposited in the PDB (PDB ID 5UV4).

\section{AUTHOR CONTRIBUTIONS}

BA and PA conceived the project; BA executed all the experiments; NV, helped identify the drought stress response of $Z m S I R K 1$. RC helped prepare the protein crystal, collected the

\section{REFERENCES}

Albert, M., Jehle, A. K., Mueller, K., Eisele, C., Lipschis, M., and Felix, G. (2010). Arabidopsis thaliana pattern recognition receptors for bacterial elongation factor Tu and flagellin can be combined to form functional chimeric receptors. J. Biol. Chem. 285, 19035-19042. doi: 10.1074/jbc.M110.124800

Apsel, B., Blair, J. A., Gonzalez, B., Nazif, T. M., Feldman, M. E., Aizenstein, B., et al. (2008). Targeted polypharmacology: discovery of dual inhibitors of tyrosine and phosphoinositide kinases. Nat. Chem. Biol. 4, 691-699. doi: 10.1038/ nchembio. 117

Arrowsmith, C. H., Audia, J. E., Austin, C., Baell, J., Bennett, J., Blagg, J., et al. (2015). The promise and peril of chemical probes. Nat. Chem. Biol. 11, 536-541. doi: $10.1038 /$ nchembio. 1867

Bain, J., Plater, L., Elliott, M., Shpiro, N., Hastie, C. J., McLauchlan, H., et al. (2007). The selectivity of protein kinase inhibitors: a further update. Biochem. J. 408, 297-315. doi: 10.1042/BJ20070797

Blaum, B. S., Mazzotta, S., Noldeke, E. R., Halter, T., Madlung, J., Kemmerling, B., et al. (2014). Structure of the pseudokinase domain of BIR2, a regulator of BAK1-mediated immune signaling in Arabidopsis. J. Struct. Biol. 186, 112-121. doi: 10.1016/j.jsb.2014.02.005

Bojar, D., Martinez, J., Santiago, J., Rybin, V., Bayliss, R., and Hothorn, M. (2014). Crystal structures of the phosphorylated BRI1 kinase domain and implications for brassinosteroid signal initiation. Plant J. 78, 31-43. doi: 10.1111/tpj. 12445

Chen, V. B., Arendall, W. B. III., Headd, J. J., Keedy, D. A., Immormino, R. M., Kapral, G. J., et al. (2010). MolProbity: all-atom structure validation for
$\mathrm{X}$-ray diffraction data and solved the protein structure; $\mathrm{RC}$ and $\mathrm{BA}$ analyzed the structural data; BA, RC, and PA wrote the paper. $\mathrm{KM}, \mathrm{OG}$, and PA helped coordinate the project. LF performed the phylogenetic analyses. All authors reviewed the manuscript.

\section{FUNDING}

SGC, a registered charity (number 1097737) that receives funds from AbbVie, Bayer Pharma AG, Boehringer Ingelheim, the Canada Foundation for Innovation, Genome Canada, GlaxoSmithKline, Janssen, Lilly Canada, Merck \& Co., the Novartis Research Foundation, the Ontario Ministry of Economic Development and Innovation, Pfizer, the São Paulo Research Foundation (FAPESP grant 13/50724-5), Takeda, EU/EFPIA Innovative Medicines Initiative (IMI) Joint Undertaking (ULTRA-DD grant 115766) and the Wellcome Trust (092809/Z/10/Z).

\section{ACKNOWLEDGMENTS}

This work used NE-CAT beamline 24-ID-C (GM103403) and a Pilatus detector (RR029205) at the Advanced Photon Source (DE-AC02-06CH11357); we thank beamline scientist Dr. Frank V. Murphy for assistance during data collection. PA is a $\mathrm{CNPq}$ productivity research fellow.

\section{SUPPLEMENTARY MATERIAL}

The Supplementary Material for this article can be found online at: http://journal.frontiersin.org/article/10.3389/fpls.2017.00852/ full\#supplementary-material

macromolecular crystallography. Acta Crystallogr. D Biol. Crystallogr. 66(Pt 1), 12-21. doi: 10.1107/S0907444909042073

Cheng, H., Addona, T., Keshishian, H., Dahlstrand, E., Lu, C., Dorsch, M., et al. (2007). Regulation of IRAK-4 kinase activity via autophosphorylation within its activation loop. Biochem. Biophys. Res. Commun. 352, 609-616. doi: 10.1016/j. bbrc.2006.11.068

Comai, L. (2005). The advantages and disadvantages of being polyploid. Nat. Rev. Genet. 6, 836-846. doi: 10.1038/nrg1711

Cowtan, K. (2006). The Buccaneer software for automated model building. 1. Tracing protein chains. Acta Crystallogr. D Biol. Crystallogr. 62(Pt 9), 1002-1011. doi: 10.1107/S0907444906022116

De Bondt, H. L., Rosenblatt, J., Jancarik, J., Jones, H. D., Morgan, D. O., and Kim, S. H. (1993). Crystal structure of cyclin-dependent kinase 2. Nature 363, 595-602. doi: 10.1038/363595a0

De Rybel, B., Audenaert, D., Vert, G., Rozhon, W., Mayerhofer, J., Peelman, F., et al. (2009). Chemical inhibition of a subset of Arabidopsis thaliana GSK3-like kinases activates brassinosteroid signaling. Chem. Biol. 16, 594-604. doi: 10.1016/j.chembiol.2009.04.008

De Smet, I., Voss, U., Jurgens, G., and Beeckman, T. (2009). Receptor-like kinases shape the plant. Nat. Cell Biol. 11, 1166-1173. doi: 10.1038/ncb1009-1166

Dodds, P. N., and Rathjen, J. P. (2010). Plant immunity: towards an integrated view of plant-pathogen interactions. Nat. Rev. Genet. 11, 539-548. doi: 10.1038/ $\operatorname{nrg} 2812$

Emsley, P., Lohkamp, B., Scott, W. G., and Cowtan, K. (2010). Features and development of Coot. Acta Crystallogr. D Biol. Crystallogr. 66(Pt 4), 486-501. doi: 10.1107/S0907444910007493 
Gorrec, F. (2015). The MORPHEUS II protein crystallization screen. Acta Crystallogr. F Struct. Biol. Commun. 71(Pt 7), 831-837. doi: 10.1107/ S2053230X1500967X

Grutter, C., Sreeramulu, S., Sessa, G., and Rauh, D. (2013). Structural characterization of the RLCK family member BSK8: a pseudokinase with an unprecedented architecture. J. Mol. Biol. 425, 4455-4467. doi: 10.1016/j.jmb. 2013.07.034

Hantschel, O., and Superti-Furga, G. (2004). Regulation of the c-Abl and Bcr-Abl tyrosine kinases. Nat. Rev. Mol. Cell Biol. 5, 33-44. doi: 10.1038/nrm1280

Holm, L., and Rosenstrom, P. (2010). Dali server: conservation mapping in 3D. Nucleic Acids Res. 38, W545-W549. doi: 10.1093/nar/gkq366

Kabsch, W. (2010). XDS. Acta Crystallogr. D Biol. Crystallogr. 66(Pt 2), 125-132. doi: 10.1107/S0907444909047337

Karplus, P. A., and Diederichs, K. (2012). Linking crystallographic model and data quality. Science 336, 1030-1033. doi: 10.1126/science.1218231

Kornev, A. P., Haste, N. M., Taylor, S. S., and Eyck, L. F. (2006). Surface comparison of active and inactive protein kinases identifies a conserved activation mechanism. Proc. Natl. Acad. Sci. U.S.A. 103, 17783-17788. doi: $10.1073 /$ pnas.0607656103

Kuglstatter, A., Villasenor, A. G., Shaw, D., Lee, S. W., Tsing, S., Niu, L., et al. (2007). Cutting edge: IL-1 receptor-associated kinase 4 structures reveal novel features and multiple conformations. J. Immunol. 178, 2641-2645.

Laemmli, U. K. (1970). Cleavage of structural proteins during the assembly of the head of bacteriophage T4. Nature 227, 680-685.

Levinson, N. M., Kuchment, O., Shen, K., Young, M. A., Koldobskiy, M., Karplus, M., et al. (2006). A Src-like inactive conformation in the abl tyrosine kinase domain. PLoS Biol. 4:e144. doi: 10.1371/journal.pbio.004 0144

McCoy, A. J., Grosse-Kunstleve, R. W., Adams, P. D., Winn, M. D., Storoni, L. C., and Read, R. J. (2007). Phaser crystallographic software. J. Appl. Crystallogr. 40(Pt 4), 658-674. doi: 10.1107/S0021889807021206

Niesen, F. H., Berglund, H., and Vedadi, M. (2007). The use of differential scanning fluorimetry to detect ligand interactions that promote protein stability. Nat. Protoc. 2, 2212-2221. doi: 10.1038/nprot.2007.321

Niittyla, T., Fuglsang, A. T., Palmgren, M. G., Frommer, W. B., and Schulze, W. X. (2007). Temporal analysis of sucrose-induced phosphorylation changes in plasma membrane proteins of Arabidopsis. Mol. Cell. Proteomics 6, 1711-1726. doi: 10.1074/mcp.M700164-MCP200

Savitsky, P., Bray, J., Cooper, C. D., Marsden, B. D., Mahajan, P., BurgessBrown, N. A., et al. (2010). High-throughput production of human proteins for crystallization: the SGC experience. J. Struct. Biol. 172, 3-13. doi: 10.1016/j. jsb.2010.06.008

Schindler, T., Sicheri, F., Pico, A., Gazit, A., Levitzki, A., and Kuriyan, J. (1999). Crystal structure of Hck in complex with a Src family-selective tyrosine kinase inhibitor. Mol. Cell 3, 639-648.

Schulze-Gahmen, U., De Bondt, H. L., and Kim, S. H. (1996). High-resolution crystal structures of human cyclin-dependent kinase 2 with and without ATP: bound waters and natural ligand as guides for inhibitor design. J. Med. Chem. 39, 4540-4546. doi: 10.1021/jm960402a

Sekhon, R. S., Lin, H., Childs, K. L., Hansey, C. N., Buell, C. R., de Leon, N., et al. (2011). Genome-wide atlas of transcription during maize development. Plant J. 66, 553-563. doi: 10.1111/j.1365-313X.2011.04527.x

Shiu, S. H., and Bleecker, A. B. (2001). Receptor-like kinases from Arabidopsis form a monophyletic gene family related to animal receptor kinases. Proc. Natl. Acad. Sci. U.S.A. 98, 10763-10768. doi: 10.1073/pnas.181141598
Shortt, J., Ott, C. J., Johnstone, R. W., and Bradner, J. E. (2017). A chemical probe toolbox for dissecting the cancer epigenome. Nat. Rev. Cancer 17, 160-183. doi: $10.1038 / \mathrm{nrc} .2016 .148$

Stelpflug, S. C., Sekhon, R. S., Vaillancourt, B., Hirsch, C. N., Buell, C. R., de Leon, N., et al. (2016). An expanded maize gene expression atlas based on RNA sequencing and its use to explore root development. Plant Genome 9. doi: 10.3835/plantgenome2015.04.0025

Tang, W., Kim, T. W., Oses-Prieto, J. A., Sun, Y., Deng, Z., Zhu, S., et al. (2008). BSKs mediate signal transduction from the receptor kinase BRI1 in Arabidopsis. Science 321, 557-560. doi: 10.1126/science.1156973

Vert, G., and Chory, J. (2006). Downstream nuclear events in brassinosteroid signalling. Nature 441, 96-100. doi: 10.1038/nature04681

Wang, X., Goshe, M. B., Soderblom, E. J., Phinney, B. S., Kuchar, J. A., Li, J., et al. (2005). Identification and functional analysis of in vivo phosphorylation sites of the Arabidopsis BRASSINOSTEROID-INSENSITIVE1 receptor kinase. Plant Cell 17, 1685-1703. doi: 10.1105/tpc.105.031393

Wang, Z., Liu, J., Sudom, A., Ayres, M., Li, S., Wesche, H., et al. (2006). Crystal structures of IRAK-4 kinase in complex with inhibitors: a serine/threonine kinase with tyrosine as a gatekeeper. Structure 14, 1835-1844. doi: 10.1016/j. str.2006.11.001

Winn, M. D., Ballard, C. C., Cowtan, K. D., Dodson, E. J., Emsley, P., Evans, P. R., et al. (2011). Overview of the CCP4 suite and current developments. Acta Crystallogr. D Biol. Crystallogr. 67(Pt 4), 235-242. doi: 10.1107/ S0907444910045749

Wu, X. N., Sanchez Rodriguez, C., Pertl-Obermeyer, H., Obermeyer, G., and Schulze, W. X. (2013). Sucrose-induced receptor kinase SIRK1 regulates a plasma membrane aquaporin in Arabidopsis. Mol. Cell. Proteomics 12, 2856-2873. doi: 10.1074/mcp.M113.029579

Xu, W., Doshi, A., Lei, M., Eck, M. J., and Harrison, S. C. (1999). Crystal structures of c-Src reveal features of its autoinhibitory mechanism. Mol. Cell 3, 629-638.

Yan, L., Ma, Y., Liu, D., Wei, X., Sun, Y., Chen, X., et al. (2012). Structural basis for the impact of phosphorylation on the activation of plant receptor-like kinase BAK1. Cell Res. 22, 1304-1308. doi: 10.1038/cr.2012.74

Yang, T., Chaudhuri, S., Yang, L., Du, L., and Poovaiah, B. W. (2010). A calcium/calmodulin-regulated member of the receptor-like kinase family confers cold tolerance in plants. J. Biol. Chem. 285, 7119-7126. doi: 10.1074/ jbc.M109.035659

Zhang, K. Y., Cowtan, K., and Main, P. (1997). Combining constraints for electrondensity modification. Methods Enzymol. 277, 53-64.

Zheng, J., Fu, J., Gou, M., Huai, J., Liu, Y., Jian, M., et al. (2010). Genome-wide transcriptome analysis of two maize inbred lines under drought stress. Plant Mol. Biol. 72, 407-421. doi: 10.1007/s11103-0099579-6

Conflict of Interest Statement: The authors declare that the research was conducted in the absence of any commercial or financial relationships that could be construed as a potential conflict of interest.

Copyright (c) 2017 Aquino, Couñago, Verza, Ferreira, Massirer, Gileadi and Arruda. This is an open-access article distributed under the terms of the Creative Commons Attribution License (CC BY). The use, distribution or reproduction in other forums is permitted, provided the original author(s) or licensor are credited and that the original publication in this journal is cited, in accordance with accepted academic practice. No use, distribution or reproduction is permitted which does not comply with these terms. 